International Journal of Pure and Applied Mathematics

Volume 109 No. $4 \quad 2016,889-897$

ISSN: $1311-8080$ (printed version); ISSN: 1314-3395 (on-line version)

url: http://www.ijpam.eu

doi: 10.12732/ijpam.v109i4.12

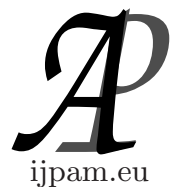

\title{
A COUPLED FIXED POINT THEOREM IN $b$-METRIC SPACES
}

\author{
Wasfi Shatanawi $^{1 \S}$, Mohammad Bani Hani ${ }^{2}$ \\ ${ }^{1}$ Department of Mathematics and General Courses \\ Prince Sultan University \\ Riyadh, SAUDI ARABIA \\ ${ }^{1}$ Department of Mathematics \\ Hashemite University \\ Zarqa, JORDAN \\ ${ }^{2}$ Department of Mathematics \\ Faculty of Science \\ Irbid National University \\ Irbid, JORDAN
}

\begin{abstract}
In this paper, we utilize the notion of coupled fixed point in sense of Bhaker and Lakshmikantham [T. Bhakar, V. Lakshmikantham, Fixed point theorems in partially orderd metric spaces and applications, Nonlinear Anal., 65 (2006), 1379-1393] to introduce and prove a coupled fixed point theorem in a $b$-metric space. Our contractive condition is the most general contractive form in linear form. Our results modified and generalized many exciting results in the literature.
\end{abstract}

AMS Subject Classification: 47H10, 54H25

Key Words: fixed point, common fixed point, $b$-metric space, quasi-contraction, metric spaces

\section{Introduction}

Bhaskar and LakshmiKantham [2] initiated the notion of coupled fixed point

Received: $\quad$ August 3, 2016

Revised: $\quad$ September 3, 2016

Published: $\quad$ October 7, 2016

${ }^{\S}$ Correspondence author (c) 2016 Academic Publications, Ltd. url: www.acadpubl.eu 
and they proved some coupled fixed point theorems in standard metric spaces. Then after, many authors formulated and studied some coupled fixed point theorems, for examples see $[2,3,4,5,6]$. The notion of $b$-metric spaces was introduced by Czerwik [1] as a generalization of standard metric spaces.

We begin with the definition of $b$-metric spaces:

Definition 1.1. [1] Let $\mathrm{X}$ be a nonempty set and $s \geq 1$ be a given real number. A function $d: X \times X \rightarrow \mathbb{R}^{+}$is a b-metric iff $\forall x, y, z \in X$ the following conditions hold

(b1) $d(x, y)=0$ iff $x=y$,

(b2) $d(x, y)=d(y, x)$,

(b3) $d(x, z) \leq s[d(x, y)+d(y, z)]$.

Definition 1.2. [7] Let $(X, d)$ be a metric space. Then the sequence $\left(x_{n}\right)$ in $\mathrm{X}$ converges to an element $x \in X$, if $\forall \epsilon>0, \exists k \in N$ such that: $d\left(x_{n}, x\right)<\epsilon, \forall n \geq k$; that is, $\lim _{n \rightarrow \infty} d\left(x_{n}, x\right)=0$.

Definition 1.3. [7] A sequence $x_{n}$ in a metric space $(X, d)$ is called a Cauchy sequence, if $\forall \epsilon>0, \exists k \in N$ such that: $d\left(x_{n}, x_{m}\right)<\epsilon, \forall m, n \geq k$; that is, $\lim _{n, m \rightarrow+\infty} d\left(x_{n}, x_{m}\right)=0$.

Definition 1.4. [7] A metric space is called complete if every Cauchy sequence converges to an element in the same metric space.

Definition 1.5. [2] An element $(\mathrm{x}, \mathrm{y}) \in X \times X$ is called a coupled fixed point of a mapping $F: X \times X \longrightarrow X$ if $F(x, y)=x$ and $F(y, x)=y$.

Definition 1.6. [4] An element $(\mathrm{x}, \mathrm{y}) \in X \times X$ is called a coupled coincidence point of the mappings $F: X \times X \longrightarrow X$ and $g: X \longrightarrow X$ if $F(x, y)=g x$ and $F(y, x)=g y$.

Definition 1.7. [4] An element $(\mathrm{x}, \mathrm{y}) \in X \times X$ is called a coupled fixed point of the mappings $F: X \times X \longrightarrow X$ and $g: X \longrightarrow X$ if $F(x, y)=g x=x$ and $F(y, x)=g y=y$.

Definition 1.8. [4] Let $X$ be a nonempty set. Then we say that the mappings $F: X \times X \longrightarrow X$ and $g: X \longrightarrow X$ are commutative if $g F(x, y)=F(g x, g y)$. 


\section{Main Results}

We start our results with the following coupled fixed point theorem.

Theorem 2.1. Let $(X, d)$ be a complete b-metric space with constant $S$ $\geq 1$. Let $F: X \times X \longrightarrow X$ and $g: X \longrightarrow X$ be two mappings on $X$. Suppose there exist nonnegative constants $\alpha_{i}: i=1,2, \ldots, 10$ such that

$$
\begin{aligned}
d(F(x, y), F(u, v)) \leq \quad & \alpha_{1} d(g x, g u)+\alpha_{2} d(g y, g v) \\
& +\alpha_{3} d(F(x, y), g x)+\alpha_{4} d(F(y, x), g y) \\
& +\alpha_{5} d(F(u, v), g u)+\alpha_{6} d(F(v, u), g v) \\
& +\alpha_{7} d(F(x, y), g u)+\alpha_{8} d(F(y, x), g v) \\
& +\alpha_{9} d(F(u, v), g x)+\alpha_{10} d(F(v, u), g y),
\end{aligned}
$$

holds for all $x, y, u, v \in X$. Also suppose the following conditions:

i) $s \alpha_{1}+s \alpha_{2}+s \alpha_{3}+s \alpha_{4}+\alpha_{5}+\alpha_{6}+\alpha_{7}+\alpha_{8}+\left(s^{2}+s\right) \alpha_{9}+\left(s^{2}+s\right) \alpha_{10}<1$.

ii) $g(X)$ is a complete subspace of $X$.

iii) $F(X \times X) \subseteq g(X)$.

Then $F$ and $g$ have a coupled coincidence point. In addition, if $F$ and $g$ are weakly compatible, then $F$ and $g$ have a unique coupled fixed point. Moreover, for any $x_{1} \in X$, the sequence $\left\{x_{n}\right\}$ defined by $g\left(x_{n+1}\right)=F\left(x_{n}, x_{n}\right)$ converges to the common fixed point of $F$ and $g$.

Proof. Let $x_{0} \in X$. Since $F(X \times X) \subseteq g(X)$, then $\exists x_{1} \in X$ such that $F\left(x_{0}, x_{0}\right)=g x_{1}$. Again, since $F(X \times X) \subseteq g(X)$, then $\exists x_{2} \in X$ such that $F\left(x_{1}, x_{1}\right)=g x_{2}$. Continuing this process we obtain a sequence $\left(y_{n}\right)$ in $g(X)$, such that

$$
y_{n}=g x_{n+1}=F\left(x_{n}, x_{n}\right)
$$

Now, we have

$$
\begin{aligned}
d\left(g x_{n+1}, g x_{n+2}\right) & =d\left(F\left(x_{n}, x_{n}\right), F\left(x_{n+1}, x_{n+1}\right)\right) \\
& \leq \alpha_{1} d\left(g x_{n}, g x_{n+1}\right)+\alpha_{2} d\left(g x_{n}, g x_{n+1}\right) \\
& +\alpha_{3} d\left(F\left(x_{n}, x_{n}\right), g x_{n}\right)+\alpha_{4} d\left(F\left(x_{n}, x_{n}\right), g x_{n}\right) \\
& +\alpha_{5} d\left(F\left(x_{n+1}, x_{n+1}\right), g x_{n+1}\right)+\alpha_{6} d\left(F\left(x_{n+1}, x_{n+1}\right), g x_{n+1}\right) \\
& +\alpha_{7} d\left(F\left(x_{n}, x_{n}\right), g x_{n+1}\right)+\alpha_{8} d\left(F\left(x_{n}, x_{n}\right), g x_{n+1}\right) \\
& +\alpha_{9} d\left(F\left(x_{n+1}, x_{n+1}\right), g x_{n}\right)+\alpha_{10} d\left(F\left(x_{n+1}, x_{n+1}\right), g x_{n}\right) .
\end{aligned}
$$


Therefore

$$
\begin{aligned}
d\left(g x_{n+1}, g x_{n+2}\right) \leq & \alpha_{1} d\left(g x_{n}, g x_{n+1}\right)+\alpha_{2} d\left(g x_{n}, g x_{n+1}\right) \\
& +\alpha_{3} d\left(g x_{n+1}, g x_{n}\right)+\alpha_{4} d\left(g x_{n+1}, g x_{n}\right) \\
& +\alpha_{5} d\left(g x_{n+2}, g x_{n+1}\right)+\alpha_{6} d\left(g x_{n+2}, g x_{n+1}\right) \\
& +\alpha_{7} d\left(g x_{n+1}, g x_{n+1}\right)+\alpha_{8} d\left(g x_{n+1}, g x_{n+1}\right) \\
& +\alpha_{9} d\left(g x_{n+2}, g x_{n}\right)+\alpha_{10} d\left(g x_{n+2}, g x_{n}\right) \\
= & \left(\alpha_{1}+\alpha_{2}+\alpha_{3}+\alpha_{4}\right) d\left(g x_{n}, g x_{n+1}\right) \\
& +\left(\alpha_{5}+\alpha_{6}\right) d\left(g x_{n+1}, g x_{n+2}\right) \\
& +\left(\alpha_{9}+\alpha_{10}\right) d\left(g x_{n}, g x_{n+2}\right)
\end{aligned}
$$

which implies that

$$
\begin{aligned}
{\left[1-\alpha_{5}-\alpha_{6}\right] d\left(g x_{n+1}, g x_{n+2}\right) \leq\left(\alpha_{9}+\right.} & \left.\alpha_{10}\right) d\left(g x_{n}, g x_{n+2}\right) \\
& +\left(\alpha_{1}+\alpha_{2}+\alpha_{3}+\alpha_{4}\right) d\left(g x_{n}, g x_{n+1}\right) .
\end{aligned}
$$

Since

$$
d\left(g x_{n}, g x_{n+2}\right) \leq s d\left(g x_{n}, g x_{n+1}\right)+s d\left(g x_{n+1}, g x_{n+2}\right),
$$

we have

$$
\begin{aligned}
{\left[1-\alpha_{5}-\alpha_{6}\right] d\left(g x_{n+1}, g x_{n+2}\right) \leq s\left(\alpha_{9}+\alpha_{10}\right)\left[d\left(g x_{n}, g x_{n+1}\right)+d\left(g x_{n+1}, g x_{n+2}\right)\right] } \\
+\left(\alpha_{1}+\alpha_{2}+\alpha_{3}+\alpha_{4}\right) d\left(g x_{n}, g x_{n+1}\right)
\end{aligned}
$$

or

$$
\begin{aligned}
& {\left[1-\alpha_{5}-\alpha_{6}-s \alpha_{9}-s \alpha_{10}\right] d\left(g x_{n+1}, g x_{n+2}\right)} \\
& \leq\left(\alpha_{1}+\alpha_{2}+\alpha_{3}+\alpha_{4}+s \alpha_{9}+s \alpha_{10}\right) d\left(g x_{n}, g x_{n+1}\right) .
\end{aligned}
$$

Therefore, we obtain

$$
d\left(g x_{n+1}, g x_{n+2}\right) \leq \frac{\alpha_{1}+\alpha_{2}+\alpha_{3}+\alpha_{4}+s \alpha_{9}+s \alpha_{10}}{\left[1-\alpha_{5}-\alpha_{6}-s \alpha_{9}-s \alpha_{10}\right]} d\left(g x_{n}, g x_{n+1}\right) .
$$

Let us set

$$
r=\frac{\left(\alpha_{1}+\alpha_{2}+\alpha_{3}+\alpha_{4}+s \alpha_{9}+s \alpha_{10}\right)}{\left(1-\alpha_{5}-\alpha_{6}-s \alpha_{9}-s \alpha_{10}\right)}
$$

Since

$$
s r=\frac{\left(s \alpha_{1}+s \alpha_{2}+s \alpha_{3}+s \alpha_{4}+s^{2} \alpha_{9}+s^{2} \alpha_{10}\right)}{\left(1-\alpha_{5}-\alpha_{6}-s \alpha_{9}-s \alpha_{10}\right)}<1
$$


and $r \leq s r$, we get $r<1$, then

$$
d\left(g x_{n+1}, g x_{n+2}\right) \leq r d\left(g x_{n}, g x_{n+1}\right)
$$

Again,

$$
\begin{aligned}
d\left(g x_{n}, g x_{n+1}\right)= & d\left(F\left(x_{n-1}, x_{n-1}\right), F\left(x_{n}, x_{n}\right)\right) \\
& \leq \alpha_{1} d\left(g x_{n-1}, g x_{n}\right)+\alpha_{2} d\left(g x_{n-1}, g x_{n}\right) \\
& +\alpha_{3} d\left(F\left(x_{n-1}, x_{n-1}\right), g x_{n-1}\right)+\alpha_{4} d\left(F\left(x_{n-1}, x_{n-1}\right), g x_{n-1}\right) \\
& +\alpha_{5} d\left(F\left(x_{n}, x_{n}\right), g x_{n}\right)+\alpha_{6} d\left(F\left(x_{n}, x_{n}\right), g x_{n}\right) \\
& +\alpha_{7} d\left(F\left(x_{n-1}, x_{n-1}\right), g x_{n}\right)+\alpha_{8} d\left(F\left(x_{n-1}, x_{n-1}\right), g x_{n}\right) \\
& +\alpha_{9} d\left(F\left(x_{n}, x_{n}\right), g x_{n-1}\right)+\alpha_{10} d\left(F\left(x_{n}, x_{n}\right), g x_{n-1}\right) .
\end{aligned}
$$

So, we get

$$
\begin{aligned}
d\left(g x_{n}, g x_{n+1}\right) \leq & \alpha_{1} d\left(g x_{n-1}, g x_{n}\right)+\alpha_{2} d\left(g x_{n-1}, g x_{n}\right) \\
& +\alpha_{3} d\left(g x_{n}, g x_{n-1}\right)+\alpha_{4} d\left(g x_{n}, g x_{n-1}\right) \\
& +\alpha_{5} d\left(g x_{n+1}, g x_{n}\right)+\alpha_{6} d\left(g x_{n+1}, g x_{n}\right) \\
& +\alpha_{7} d\left(g x_{n}, g x_{n}\right)+\alpha_{8} d\left(g x_{n}, g x_{n}\right) \\
& +\alpha_{9} d\left(g x_{n+1}, g x_{n-1}\right)+\alpha_{10} d\left(g x_{n+1}, g x_{n-1}\right),
\end{aligned}
$$

which leads us to

$$
d\left(g x_{n}, g x_{n+1}\right) \leq r d\left(g x_{n-1}, g x_{n}\right) .
$$

Repeating (1) and (2), n times, we get

$$
\begin{aligned}
d\left(g x_{n+1}, g x_{n+2}\right) & \leq r^{2} d\left(g x_{n-1}, g x_{n}\right) \\
& \vdots \\
& \leq r^{n+1} d\left(g x_{0}, g x_{1}\right)
\end{aligned}
$$

Given $n, m \in N$, with $m>n$. Then

$$
\begin{aligned}
d\left(g x_{n}, g x_{m}\right) \leq & s d\left(g x_{n}, g x_{n+1}\right)+s d\left(g x_{n+1}, g x_{m}\right) \\
\leq & s d\left(g x_{n}, g x_{n+1}\right)+s^{2} d\left(g x_{n+1}, g x_{n+2}\right)+s^{2} d\left(g x_{n+2}, g x_{m}\right) \\
\leq & s d\left(g x_{n}, g x_{n+1}\right)+s^{2} d\left(g x_{n+1}, g x_{n+2}\right)+\cdots \\
& +s^{m-n} d\left(g x_{m-1}, g x_{m}\right) .
\end{aligned}
$$

From (3), we get

$$
d\left(g x_{n}, g x_{m}\right) \leq s r^{n} d\left(g x_{0}, g x_{1}\right)+s r^{n+1} d\left(g x_{0}, g x_{1}\right)
$$




$$
\begin{aligned}
& +\ldots+s^{m-n} r^{m-1} d\left(g x_{0}, g x_{1}\right) \\
\leq & s r^{n} d\left(g x_{0}, g x_{1}\right)\left[1+s r+s^{2} r^{2}+s^{3} r^{3}+\ldots\right] \\
\leq & s r^{n} d\left(g x_{0}, g x_{1}\right) \sum_{i=0}^{+\infty}(s r)^{i} \\
= & \frac{s r^{n}}{1-s r} d\left(g x_{0}, g x_{1}\right) .
\end{aligned}
$$

Since $r^{n} \longrightarrow 0$ as $n \longrightarrow+\infty$, we get that

$$
\lim _{n, m \longrightarrow+\infty} d\left(g x_{n}, g x_{m}\right)=0 .
$$

So $\left(g x_{n}\right)$ is a b-Cauchy sequence in $(g(X), d)$. By completeness of $g(X) \exists$ $\mathrm{p} \in g(X)$ such that

$$
\lim _{n \longrightarrow+\infty} g x_{n}=g u=p .
$$

We shall show that $(u, u)$ is a coupled coincidence point of $F$ and $g$.

$$
\begin{aligned}
d\left(g x_{n+1}, F(u, u)\right) & =d\left(F\left(x_{n}, x_{n}\right), F(u, u)\right) \\
& \leq \alpha_{1} d\left(g x_{n}, g u\right)+\alpha_{2} d\left(g x_{n}, g u\right) \\
& +\alpha_{3} d\left(F\left(x_{n}, x_{n}\right), g x_{n}\right)+\alpha_{4} d\left(F\left(x_{n}, x_{n}\right), g x_{n}\right) \\
& +\alpha_{5} d(F(u, u), g u)+\alpha_{6} d(F(u, u), g u) \\
& +\alpha_{7} d\left(F\left(x_{n}, x_{n}\right), g u\right)+\alpha_{8} d\left(F\left(x_{n}, x_{n}\right), g u\right) \\
& +\alpha_{9} d\left(F(u, u), g x_{n}\right)+\alpha_{10} d\left(F(u, u), g x_{n}\right),
\end{aligned}
$$

which implies that

$$
\begin{aligned}
d\left(g x_{n+1}, F(u, u)\right) & \leq \alpha_{1} d\left(g x_{n}, g u\right)+\alpha_{2} d\left(g x_{n}, g u\right) \\
& +\alpha_{3} d\left(g x_{n+1}, g x_{n}\right)+\alpha_{4} d\left(g x_{n+1}, g x_{n}\right) \\
& +\alpha_{5} d(F(u, u), g u)+\alpha_{6} d(F(u, u), g u) \\
& +\alpha_{7} d\left(g x_{n+1}, g u\right)+\alpha_{8} d\left(g x_{n+1}, g u\right) \\
& +\alpha_{9} d\left(F(u, u), g x_{n}\right)+\alpha_{10} d\left(F(u, u), g x_{n}\right) .
\end{aligned}
$$

Note that

$$
d(g u, F(u, u)) \leq s d\left(\left(g u, g x_{n+1}\right)+d\left(g x_{n+1}, F(u, u)\right)\right) .
$$

Letting $n \longrightarrow+\infty$ in above inequality we get

$$
\frac{1}{s} d(g u, F(u, u)) \leq \lim _{n \rightarrow \infty} d\left(g x_{n+1}, F(u, u)\right) .
$$

Also, 


$$
d\left(g x_{n+1}, g x_{n}\right) \leq s\left(d\left(g x_{n+1}, g u\right)+d\left(g u, g x_{n}\right)\right) .
$$

Letting $n \longrightarrow+\infty$ in above inequality we get

$$
\lim _{n \longrightarrow \infty}\left(g x_{n+1}, g x_{n}\right)=0 .
$$

Again, note that

$$
d\left(F(u, u), g x_{n}\right) \leq s\left(d(F(u, u), g u)+d\left(g u, g x_{n}\right)\right)
$$

Letting $n \longrightarrow+\infty$ in above inequality, we get

$$
\lim _{n \rightarrow \infty} d\left(F(u, u), g x_{n}\right) \leq s d(F(u, u), g u) .
$$

Letting $n \longrightarrow+\infty$ in (4) and using (5), (6) and (7), we get

$$
\begin{aligned}
\frac{1}{s} d(g u, F(u, u)) & \leq \alpha_{5} d(F(u, u), g u)+\alpha_{6} d(F(u, u), g u) \\
& +s \alpha_{9} d(F(u, u), g u)+s \alpha_{10} d(F(u, u), g u)
\end{aligned}
$$

which implies that

$$
d(g u, F(u, u)) \leq\left(s \alpha_{5}+s \alpha_{6}+s^{2} \alpha_{9}+s^{2} \alpha_{10}\right) d(F(u, u), g u) .
$$

Since $\left(s \alpha_{5}+s \alpha_{6}+s^{2} \alpha_{9}+s^{2} \alpha_{10}<1\right)$, we get $d(g u, F(u, u))=0$.

Hence $g u=F(u, u)$.

Hence $(u, u)$ is a coupled coincidence point of $F$ and $g$.

To prove the uniqueness of the coupled coincidence point $(u, u)$, assume that $(v, v)$ is a coupled coincidence point of $F$ and $g$. Then

$$
\begin{aligned}
d(g u, g v) & =d(F(u, u), F(v, v)) \\
& \leq \alpha_{1} d(g u, g v)+\alpha_{2} d(g u, g v) \\
& +\alpha_{3} d(F(u, u), g u)+\alpha_{4} d(F(u, u), g u) \\
& +\alpha_{5} d(F(v, v), g v)+\alpha_{6} d(F(v, v), g v) \\
& +\alpha_{7} d(F(u, u), g v)+\alpha_{8} d(F(u, u), g v) \\
& +\alpha_{9} d(F(v, v), g u)+\alpha_{10} d(F(v, v), g u)
\end{aligned}
$$

which implies that

$d(g u, g v) \leq\left(\alpha_{1}+\alpha_{2}+\alpha_{7}+\alpha_{8}+\alpha_{9}+\alpha_{10}\right) d(g u, g v)$.

Since $\left(\alpha_{1}+\alpha_{2}+\alpha_{7}+\alpha_{8}+\alpha_{9}+\alpha_{10}\right)<1$, then

$$
d(g u, g v)=0 \text {. So } g u=g v \text {. }
$$

Hence $(u, u)$ is the unique coupled coincidence point of $F$ and $g$.

Now, suppose that $F$ and $g$ are weakly compatible. Then

$g(F(u, u))=F(g u, g u)$.

Put $w=g u$. Then 


$$
\begin{aligned}
g w=g(g u) & =g(F(u, u)) \\
& =F(g u, g u) \quad \text { So, }(w, w) \text { is a coupled coincidence point of } \\
& =F(w, w) . \quad \text {. }
\end{aligned}
$$

$F$ and $g$. Therefore $g w=g u$.

Hance $g w=w$ and $F(w, w)=g w=w$.

So $(w, w)$ is a coupled fixed point of $F$ and $g$.

Corollary 2.1. Let $(X, d)$ be a complete b-metric space with constant $s$ $\geq 1$. Let $F: X \times X \longrightarrow X$ be a mapping. Suppose there exist non-negative constants $\alpha_{i}: i=1,2, \ldots, 10$ such that

$$
\begin{aligned}
d(F(x, y), F(u, v)) \leq & \alpha_{1} d(x, u)+\alpha_{2} d(y, v) \\
& +\alpha_{3} d(F(x, y), x)+\alpha_{4} d(F(y, x), y) \\
& +\alpha_{5} d(F(u, v), u)+\alpha_{6} d(F(v, u), v) \\
& +\alpha_{7} d(F(x, y), u)+\alpha_{8} d(F(y, x), v) \\
& +\alpha_{9} d(F(u, v), x)+\alpha_{10} d(F(v, u), y) .
\end{aligned}
$$

holds for all $x, y, u, v \in X$.

If $s \alpha_{1}+s \alpha_{2}+s \alpha_{3}+s \alpha_{4}+\alpha_{5}+\alpha_{6}+\alpha_{7}+\alpha_{8}+\left(s^{2}+s\right) \alpha_{9}+\left(s^{2}+s\right) \alpha_{10}<1$, then $F$ has a unique coupled fixed point.

Proof. It follows from Theorem 2.1 by taking $g=\operatorname{Id}_{\mathrm{x}}$ the identity mapping.

Corollary 2.2. Let $(X, d)$ be a complete metric space. Let $F: X \times X \longrightarrow$ $X$ be a mapping. Suppose there exist non-negative constants $\alpha_{i}: i=1,2, \ldots, 10$ such that

$$
\begin{aligned}
d(F(x, y), F(u, v)) \leq & \alpha_{1} d(x, u)+\alpha_{2} d(y, v) \\
& +\alpha_{3} d(F(x, y), x)+\alpha_{4} d(F(y, x), y) \\
& +\alpha_{5} d(F(u, v), u)+\alpha_{6} d(F(v, u), v) \\
& +\alpha_{7} d(F(x, y), u)+\alpha_{8} d(F(y, x), v) \\
& +\alpha_{9} d(F(u, v), x)+\alpha_{10} d(F(v, u), y) .
\end{aligned}
$$

holds for all $x, y, u, v \in X$.

If $\alpha_{1}+\alpha_{2}+\alpha_{3}+\alpha_{4}+\alpha_{5}+\alpha_{6}+\alpha_{7}+\alpha_{8}+2 \alpha_{9}+2 \alpha_{10}<1$, then $F$ has a unique coupled fixed point.

Proof. It follows from Corollary 2.1 by noting that $d$ is a $b$-metric space with $s=1$. 


\section{References}

[1] S. Ćzerwik, Nonliner set-valud contraction mappings in b-metric spaces, Atti Semin. Mat. Fis. Univ. Modena, 46, No. 2 (1998), 263-276.

[2] T. Bhakar, V. Lakshmikantham, Fixed point theorems in partially orderd metric spaces and applications, Nonlinear Anal., 65 (2006), 1379-1393.

[3] Y.J. Cho, Z. Kadelburg, R. Saadati, W. Shatanawi, Coupled fixed point theorems under weak contractions, Discrete Dynamics in Nature and Society (2012).

[4] V. Lakshmikantham, L.B. Ćirić, Coupled fixed point theorems for nonlinear contractions in partially ordered metric spaces, Nonlinear Anal., 70 (2009).

[5] W. Shatanawi, On w-compatible mappings and common coupled coincidence point in cone metric spaces, Applied Mathematics Letters, 25, No. 6, 925-931.

[6] W. Shatanawi, Partially ordered cone metric spaces and coupled fixed point results, Computers and Mathematics with Applications, 60 (2010), 2508-2515.

[7] M. Boriceanu, M. Bota, A. Pertrusel, Multivaled fractals in b-metric space, Cent. Eur. J. Math., 8 (2010), 367-377. 
